\title{
The Application and Research of Humanistic Care in Operation Room
}

\author{
Yanjie Han \\ Harbin Medical University Cancer Hospital, Harbin 150000, Heilongjiang, China \\ Email: hanyanjie2000@163.com
}

\begin{abstract}
Objective - To conduct studies on the application effect of humanistic care in the operation room. Methods A total of 90 patients who received surgical treatment in our hospital from January 2021 to May 2021 were selected and divided into experimental group and control group based on the order of admission, with 45 patients in each group. The patients in the control group were treated with routine surgical nursing, and the patients in the experimental group were treated with humanistic care on the basis of routine surgical nursing. Comparison was made in the application effect of the two groups. Results - The scores of Self-Rating Depression Scale and Self-Rating Anxiety Scale in two groups demonstrated significant decreasing. The scores of the Self-Rating Depression Scale and Self-Rating Anxiety Scale were significantly reduced in the two groups after psychological nursing intervention. Conclusions - Effective humanistic care can not only contributes to the relief of anxiety or depression and other unhealthy emotions of patients, but also serves as an important way to maintain a good doctor-patient relationship. Humanistic care has certain application value in clinical applications. Keywords: operation room, humanistic care, application value
\end{abstract}

The operation room is a very important medical department in hospital, whose main function is to provide rescue and surgical treatment for related patients. The operation room is a place with very strict requirements on encapsulation. It is difficult for patients to get humanistic care in the operation room. As a consequence, they are prone to anxiety, depression, fear and other adverse psychology in the face of diseases, which are very detrimental to the prognosis. Surgical treatment can help patients remove lesions and improve their condition. However, as a stress source, surgery is likely to cause secondary trauma to patients, which directly affects the prognosis and quality of life of patients. Routine nursing intervention in clinical practices can effectively reduce the incidence of postoperative complications, help patients recover faster and better, and provide guarantee for patients' recovery and quality of life. With the rapid development of economy in recent years, great changes have taken place in people's living standard and lifestyle. Both patients and their familial relatives have higher requirements for medical technology, and they pay more attention to the quality of care and humanistic care in the process of medical care with the hope that patients can get psychological support in the process of treatment with quick recovery. This paper mainly studies the application of humanistic care in the operation room to provide reference for the clinical care of humanistic care.

\section{Data and methods}

\subsection{General data}

A total of 90 patients who underwent surgical treatment in our hospital from January 2021 to May 2021 were selected and divided into experimental group and control group abased on the order of admission, with 45 patients in each group. Patients in the control group were treated with routine surgical nursing, and patients in the experimental group were treated with humanistic care on the basis of routine surgical nursing. This study has been approved by the Ethics Committee of our hospital. The patients and their family members were aware of the purpose and process of the experiment, and signed the experimental consent. In the study group, there were 23 males and 22 females, aged from 33 to 78 years old, with an average age of $(46.8 \pm 4.2)$ years old. In the control group, there were 21 males and 24 females, aged $34 \sim 77$ years old, with an average age of (47.2 \pm 4.0$)$ years old. There was no statistical difference in the basic data between the two groups (P>0.05).

\subsection{Methods}

Routine nursing was carried out for patients in the control group: Various medical checks were carried out before the operation to understand the patients' vital signs and disease conditions, and a reasonable and perfect therapeutic schedule was formulated according to the evaluation of the condition, so as to make perfect preparations for the implementation of gastric surgery. Observe the patient's emotions and psychological status, and then give effective psychological counseling to the patient. After the operation, the patient's vital signs were closely observed, the recovery of the surgical incision was 
checked from time to time, treatment and nursing were performed according to the doctor's advice, and relevant guidance was given before the patients are discharged from the hospital.

Psychological nursing was carried out on the basis of routine nursing for patients in the experimental group. (1) Set up a special psychological nursing group. Special psychological care should be given to each team member. After management training and examination, the examinee should master empathy, hint, comfort and other psychological nursing means. Strict psychological nursing procedures are established within the department. (2) Pay attention to the psychological state of patients. The physiological condition of the patient will greatly affect his psychological condition. Doctors and nurses should understand and pay attention to the dynamic changes of various physiological indexes of patients before and after operation. Close attention can be given to whether the patient show anxiety, depression tendency, in order to carry out timely and positive psychological counseling. (3) Effective communication. Effective communication with patients is an effective way to understand the psychological situation of patients and remove their bad emotions. To alleviate the anxiety of patients, we must enable patients to understand their condition comprehensively and objectively, and face up to their condition. Secondly, the various medical measures, the use and effectiveness of medical means in the medical process can be introduced in detail to the patient, so that the patient is aware of the active and effective treatment. Medical staff should strengthen the care of patients, conduct more communication with patients with heart so as to understand their needs and meet their needs. In the accompany and care, establish patients' trust for medical staff. Enhance the confidence of patients to overcome the disease, so that patients can face the disease with a positive and optimistic state of mind. (4) Psychological support. Medical staff should pay attention to the psychological problems of patients. Medical staff should be patient and careful when communicating with patients and respect and care should be given to patients. Medical staff should give patients constant encourage as friends in psychological support. In the course of treatment, solve problems for patients with a matter-of-fact attitude. Create a relaxed, pleasant and positive treatment environment for patients.

\subsection{Observation indexes}

Humanistic care can be applied not only in the operation room, but also in the process of other clinical treatments. It is beneficial to the treatment for patients and rehabilitation, and it can ensure that patients have a good attitude for receiving treatment. The Self-Rating Anxiety Scale (SAS) and Self-Rating Depression Scale (SDS) were used to compare the scores of the two groups before and after nursing. The higher the score, the more anxious or depressed the patient was.

\subsection{Statistical methods}

SPSS17.0 statistical software was used to analyze the data. The measurement data was expressed as $\mathrm{x} \pm \mathrm{s}$, and $\mathrm{t}$ test was used, while $\mathrm{x}^{2}$ test was used for the counting data. $\mathrm{P}<0.05$ was considered statistically significant.

\section{Results}

\subsection{Comparison of depression between the two groups before and after nursing care}

After humanistic care intervention, the scores of the Self-Rating Depression Scale and the Self-Rating Anxiety Scale of patients in the two groups were significantly reduced, as shown in Table 1.

Table 1. Comparison of scores of Self-Rating Depression Scale between the two groups ( $x \pm s)$

\begin{tabular}{ccccc}
\hline Groups & Control Group & Experimental group & $\mathrm{t}$ & $\mathrm{p}$ \\
\hline Pre-nursing & $47.14 \pm 5.27$ & $46.83 \pm 5.14$ & 0.152 & \\
Post- nursing & $43.16 \pm 4.12$ & $30.11 \pm 3.08$ & 8.325 & $\mathrm{P}<0.05$ \\
\hline
\end{tabular}

\subsection{Comparison of anxiety between the two groups before and after nursing care}

After the intervention of humanistic care, the scores of the Self-Rating Anxiety Scale and the Self-Rating Anxiety Scale of patients in the two groups were significantly reduced, as shown in Table 2.

Table 2. Comparison of scores of Self-Rating Anxiety Scale between the two groups $(x \pm s)$

\begin{tabular}{ccccc}
\hline Groups & Control Group & Experimental Group & $\mathrm{t}$ & $\mathrm{p}$ \\
\hline Pre-nursing & $49.12 \pm 4.21$ & $48.65 \pm 3.96$ & 0.123 & \\
Post-nursing & $42.33 \pm 4.22$ & $38.12 \pm 5.32$ & 7.524 & $\mathrm{P}<0.05$ \\
\hline
\end{tabular}




\section{Discussion}

With the rapid development of economy in recent years, people's living standard and lifestyle have undergone tremendous changes. Both patients and their families have higher requirements for medical technology, and pay more attention to the quality of nursing and humanistic care in the process of medical care. Routine nursing intervention in clinical practice can effectively reduce the incidence of postoperative complications, help patients recover faster and better, and provide guarantee for patients' recovery and quality of life. But at the same time, the patients and their family members hope that the patient can get psychological support in the treatment process with quick recovery. The operation room is a very important department in hospital, whose main function is to provide rescue and surgical treatment for related patients. Surgical treatment can help patients remove lesions and improve their condition. But as a stress source, surgery is likely to cause secondary trauma to patients, which directly affects the prognosis and quality of life of patients.

The operation room is a place with very strict requirements for encapsulation. It is difficult for patients to get humanistic care in the operation room, and they are prone to anxiety, depression, fear and other adverse psychology in the face of diseases, which are very detrimental to the prognosis. The so-called humanistic care refers to providing patients with a humanized service and treatment environment before and after surgery and during the process, so that they feel comfortable, warm, comfortable physically and mentally. Effective humanistic care needs to be done before surgery. Detailed introduction to the relevant precautions of the operation can be made for the patient. The surgeon's rich clinical experience can be told to the patient to enhance the patient's confidence. This method can not only improve the patient's cognition and effectively relieve anxiety, but also improve the patient's sense of dependence and trust on the medical staff. During the operation, medical staff should patiently observe the patient's emotional changes, care about the patient's internal feelings, give them appropriate and timely positive encouragement, and guide the patient to cooperate with the doctor to complete the operation treatment. After the operation is completed, the patient should be informed of the results of the operation to calm down the patient's mood. In addition, careful handover should be carried out with the medical staff in the ward. In the follow-up, attention should be paid to the ward rounds and the changes of the patient's condition, so as to ensure the patient's physical recovery and mental health. Humanistic care can be applied not only in the operating room, but also in other clinical treatment processes. It can not only be conducive to the treatment and rehabilitation of patients, but also ensure that patients have a good attitude towards medical treatment, which is conducive to maintaining a harmonious doctor-patient relationship and jointly creating a harmonious and stable medical environment.

\section{Conclusion}

For patients with surgical treatment, humanistic care is of great significance to their rehabilitation and quality of life. Effective humanistic care not only can help relieve the anxiety or depression and other bad emotions of patients, but also is an important way to maintain a good doctor-patient relationship. Therefore, humanistic care has certain application value in clinical practices.

\section{References}

[1] Xin Zehong, Jiang Rui, Yin Yuexia.Application and experience of humanistic care in quality nursing service in operation room[J]. Healthy People. 2016; (11): 263-264.

[2] Sun Likai, Li Liping, He Huaying.Application of humanistic care-oriented life nursing in quality nursing service[J]. Journal of Nursing Science. 2013; (5): 21-23.

[3] Chen Hongfang.Analysis of the effect of quality nursing service on patients with malignant tumor in thoracic surgery[J]. Chinese And Foreign Medical Research. 2016; (28): 152.

[4] Lin Yanyu, Chen Chunhua, Qiu Hehua.Survey on job satisfaction of nurses in the development of quality nursing service[J]. Chinese And Foreign Medical Research. 2016; (28): 84.

[5] Fenfei. Application value of quality nursing service in patients with cirrhosis complicated with upper gastrointestinal bleeding[J]. China Journal of Pharmaceutical Economics. 2016; (10): 63.

[6] Zhuang Liping.Application of quality nursing service in the treatment of patients with abuse of psychoactive substance[J]. Pharmacy Today. 2016; (10): 64.

[7] Zhang Ting. Observation on the effect of human care for 70 patients with operations[J]. Journal of Yanan University (Medical Science Edition). 2015; (3): 79-80.

[8] Huang Lidan, Yao Hongling, Guo Xinhua.Strengthening the cultivation of humanistic care ability in the training of new employees in the operation room[J]. Journal of Clinic Nursing's Practicality. 2017; (9): 184-185. 